\title{
Health problems in survivors of childhood cancer: the need for international collaboration in long-term follow-up care
}
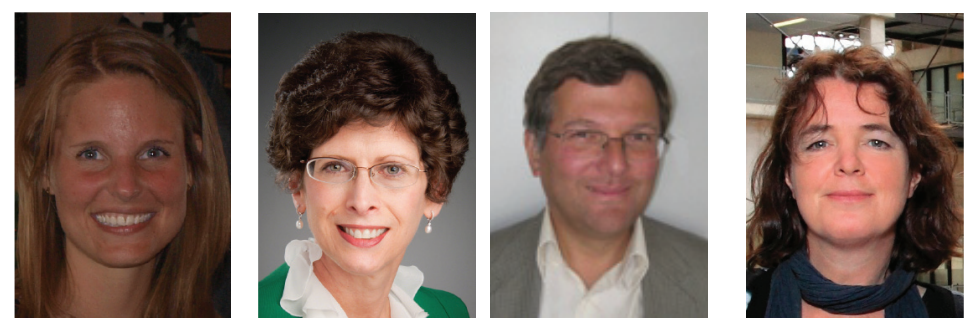

Renée L Mulder*1, Melissa M Hudson², Roderick Skinner ${ }^{3}$ \& Leontien CM Kremer

'Department of Pediatric Oncology, Emma Children's Hospital/Academic Medical Center, Amsterdam,

The Netherlands

2Departments of Oncology \& Epidemiology \& Cancer Control, St. Jude Children's Research Hospital Memphis, TN, USA

${ }^{3}$ Department of Paediatric \& Adolescent Haematology \& Oncology, Great North Children's Hospital, Royal Victoria Infirmary, Newcastle upon Tyne, UK

*Author for correspondence: Tel.. +31 205661420 - Fax: +31 206912231 =r.l.mulder@amc.uva.nl

\section{Long-term health problems in childhood cancer survivors}

Worldwide, over 200,000 children under the age of 15 years are diagnosed with cancer each year [1]. Continuing advances in the treatment of childhood cancer over the last 50 years have contributed to greatly improved survival rates. While in the 1960 s only $30 \%$ of childhood cancer patients survived 5 years or more, survival rates in developed countries have now reached $80 \%[1,2]$. As a result, most children diagnosed with cancer are expected to become long-term survivors. It is estimated that approximately one in every 680 individuals aged between 20 and 50 years is currently a survivor of a pediatric or adolescent cancer [3]. At the same time that the childhood cancer survivor population is increasing in size, it is also increasing in attained age and a significant number have reached or are entering adulthood.

"Physicians involved in the care of childhood cancer survivors

should ... provide high-quality, long-term

follow-up care based on guidelines developed by evidence-based methods."

Over the last three decades, healthcare providers, as well as survivors and their families, have become increasingly aware that successful cancer treatment may cause long-term treatment-related health problems ('late effects'), particularly among those treated during childhood when the body is still developing. These health problems may emerge many years, even decades, after the end of cancer treatment. Consequently, childhood cancer survivors are a growing group of individuals who are at high risk for premature morbidity and mortality [4]. Frequently occurring health problems affecting life expectancy and/or quality of survival include second malignancies, organ dysfunctions, endocrine and metabolic disorders, and cognitive and psychosocial impairment [5]. Previous studies have shown that approximately three out of four childhood cancer survivors develop one or more health problems, which are severe or life threatening in $25 \%$ of the cases $[6,7]$. In view of their long life expectancy, childhood cancer survivors are subject to the full spectrum of potential chronic health problems. These health problems can be expected to increase the incidence of chronic diseases in adult survivors of childhood cancer and may ultimately reduce their life expectancy $[4,5]$.

\section{Need for follow-up care for childhood cancer survivors}

Long-term follow-up care for childhood cancer survivors is important to facilitate early detection of health problems and timely initiation of interventions to preserve health. Physicians involved in the care of childhood cancer survivors should be aware of these specific health problems, counsel survivors, consult other physicians and, when available, initiate appropriate treatment.

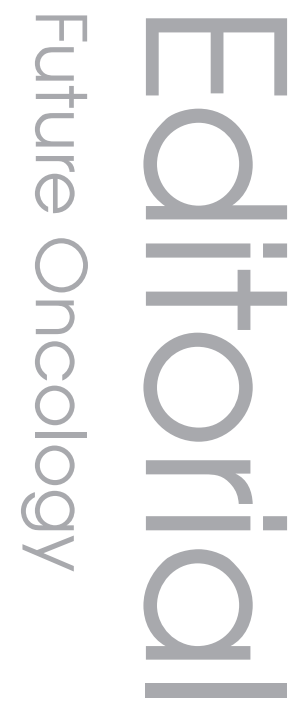
. (1) 
Therefore, in recent decades several national initiatives have started to target childhood cancer survivors for engagement in health screening and surveillance programs [8-11]. Since the 1980s, an increasing number of pediatric oncology centers have developed programs specifically for childhood cancer survivors. However, relatively few pediatric hospitals are able to provide follow-up into the adult age range [12].

\section{Need for clinical practice guidelines}

To provide high-quality care, it is essential that clinicians' knowledge is up to date. This is, however, very challenging since more than 2 million new medical papers are published each year. The number of survivorship studies has also increased substantially since 1984, quadrupling even from 1996 to 2011 [13]. Clinicians are increasingly barraged with a large volume of evidence, which is rarely summarized and is often of uncertain value. It is now recognized that several steps are needed to translate research findings into clinical practice. These steps include synthesizing the evidence into systematic reviews, developing clinical policy from evidence into evidence-based practice guidelines and applying the guidelines [14].

$$
\begin{aligned}
& \text { "Guidelines recommending proven, } \\
& \text { effective interventions and } \\
& \text { discouraging ineffective ones may } \\
& \text { reduce morbidity and mortality, and } \\
& \text { thus improve quality of life." }
\end{aligned}
$$

Guidelines can help to bridge the gap between research and clinical practice. Clinical practice guidelines are viewed as powerful tools to improve quality of care in terms of healthcare processes and health outcomes. Guidelines recommending proven, effective interventions and discouraging ineffective ones may reduce morbidity and mortality, and thus improve quality of life. Clinical practice guidelines also increase the likelihood that patients receive uniformly high-quality care, thereby reducing variability in daily healthcare practice. They can hence stimulate effective care, and facilitate communication and collaboration between different healthcare professionals, as well as between healthcare professionals and patients. In addition, clinical practice guidelines can contribute to reduced healthcare costs by increasing the efficiency of care provision and reducing unnecessary or inefficient components of health care. Access to guidelines has been also shown to reduce expenses for hospitalization, drug prescriptions, surgery and other procedures [15].

\section{Need for worldwide guidelines for surveillance of childhood cancer survivors}

As the number of long-term childhood cancer survivors has increased, the importance of understanding the needs of this unique population has also grown. To ensure that childhood and young adult cancer survivors receive optimum care, several long-term follow-up guidelines have been developed over the last 10 years by groups in North America and Europe [101-104]. Survivors and healthcare providers need guidance to be appropriately aware of and proactive about cancerand treatment-related health risks. Many studies have been published about health risks related to childhood cancer treatment. This knowledge has been used to characterize the clinical features of survivors at risk for specific health problems following specific treatments. These studies form the basis for risk-based screening and surveillance that is featured in the guidelines. Such risk-based health screening provides opportunities to enhance early detection of late health problems and initiate interventions that may preserve health and improve the quality of survivorship. However, the recommendations differ between the different guideline groups, and much work has been duplicated by developing independent guidelines without collaboration. To overcome these limitations and further improve the quality of care for childhood cancer survivors, we have initiated a worldwide collaborative endeavor to harmonize the existing guidelines for the longterm follow-up of survivors of childhood and young adult cancer: the International Late Effects of Childhood Cancer Guideline Harmonization Group (IGHG) [16]. This group works together with the European PanCareSurFup guideline working group (7th Framework Programme of the EU, 257505) to improve and harmonize recommendations for follow-up care of childhood cancer survivors. The harmonized guidelines aim to cover the full range of health problems experienced by childhood cancer survivors and provide recommendations regarding which patients need surveillance, what surveillance modalities should be used, at what frequency surveillance should be performed and what should be done when abnormalities are identified. Recommendations are graded based on the levels of available evidence.

\section{Need for implementation of guidelines \& optimal information for survivors of childhood cancer}

It is important to note that the development of evidence-based clinical practice guidelines does 
not guarantee an improvement in the quality of care. The success of guidelines depends on many factors, including their clinical context, methodology, dissemination and implementation strategies [14,15]. The IGHG will develop quality indicators to measure the adherence to the recommendations of guidelines, and the effects on important outcomes for survivors of childhood cancer.

To facilitate early detection of cancer treatment-related late effects and initiation of health-preserving interventions, survivors should actively participate in long-term followup care. For example, treatment summaries with a survivorship care plan that outlines health risks and recommendations for health screening and risk-reducing interventions can inform survivors about optimal follow-up care which is most likely to be beneficial to them. This will allow survivors to make a choice between potential benefits of screening (e.g., starting early treatment) and the potential harms (e.g., the consequences of false-positive tests results).

\section{Need for new studies in childhood cancer survivors}

Although the body of knowledge on health problems in childhood cancer survivors has increased considerably over recent decades, there are still many gaps in the evidence. To date, studies investigating the yield, risks, benefits, harms and cost-effectiveness of surveillance and treatment options for health problems related to treatment for childhood cancer are scarce. Such studies are necessary to assess whether follow-up care will improve health outcomes in survivors of childhood cancer. Evaluation should focus on diagnostic accuracy and the effect of surveillance, thereby weighing the benefits against the harms. In addition, future studies should investigate possible treatment and lifestyle intervention strategies to preserve long-term health. The ongoing work performed by the IGHG and PanCareSurFup will highlight gaps in knowledge related to survivorship care and inform the international research agenda.

\section{References}

1. Steliarova-Foucher E, Stiller C, Kaatsch P et al. Geographical patterns and time trends of cancer incidence and survival among children and adolescents in Europe since the 1970s (the ACCISproject): an epidemiological study. Lancet 364, 2097-2105 (2004).

2. SEER Cancer Statistics Review, 1975-2008. Howlader N, Noone AM, Krapcho M et al.

\section{Need for new trials in children with cancer}

Knowledge gained from late health outcomes research provides information that can guide primary prevention of health problems among newly diagnosed patients, and surveillance and secondary health-promoting interventions among long-term survivors who require specific agents and modalities to optimize disease control. Identification of treatmentrelated risk factors for health problems in survivors of childhood cancer and advancing understanding of host/genetic and behavioral factors influencing these risks will help guide clinicians and researchers as they seek to develop less toxic treatment strategies for children with cancer and investigate possible preventive interventions in childhood cancer treatment trials.

\section{Conclusion}

Childhood cancer survivors are at a high risk for health problems. Physicians involved in the care of childhood cancer survivors should be aware of these specific health problems and provide high-quality, long-term follow-up care based on guidelines developed by evidencebased methods. Survivors should be involved in clinical decision-making concerning the best available follow-up care. International collaboration between clinicians, researchers, guideline developers and survivors will contribute to optimal recommendations for follow-up care described in international guidelines and the elaboration of a research agenda for this field.

\section{Financial \& competing interests disclosure \\ The authors have no relevant affiliations or financial involvement with any organization or entity with a financial interest in or financial conflict with the sub- ject matter or materials discussed in the manuscript. This includes employment, consultancies, honoraria, stock ownership or options, expert testimony, grants or patents received or pending, or royalties. \\ No writing assistance was utilized in the production of this manuscript.}

(Eds). National Cancer Institute, MD, USA (2011).

3. Mariotto AB, Rowland JH, Yabroff KR et al. Long-Term Survivors of Childhood Cancers in the United States. Cancer Epidemiol. Biomarkers Prev. 18, 1033-1040 (2009).

4. Reulen RC, Winter DL, Frobisher C et al. Long-term cause-specific mortality among survivors of childhood cancer. JAMA 304, 172-179 (2010).
5. Late effects of childhood cancer. Wallace HB, Green DM (Eds). Arnold Publication, London, UK (2004).

6. Oeffinger KC, Mertens AC, Sklar CA et al. Chronic health conditions in adult survivors of childhood cancer. N. Engl. J. Med. 355, 1572-1582 (2006).

7. Geenen MM, Cardous-Ubbink MC, Kremer LC et al. Medical assessment of adverse health outcomes in long-term survivors of 
childhood cancer. JAMA 297, 2705-2715 (2007).

8. Nathan PC, Greenberg ML, Ness KK et al. Medical care in long-term survivors of childhood cancer: a report from the childhood cancer survivor study. J. Clin. Oncol. 26, 4401-4409 (2008).

9. Taylor A, Hawkins M, Griffiths A et al. Longterm follow-up of survivors of childhood cancer in the UK. Pediatr. Blood Cancer 42, 161-168 (2004).

10. Sieswerda E, Mulder RL, van Dijk IW et al. The EKZ/AMC childhood cancer survivor cohort: methodology, clinical characteristics, and data availability. J. Cancer Surviv. (2013) (Epub ahead of print).

11. Hudson MM, Ness KK, Nolan VG et al. Prospective medical assessment of adults surviving childhood cancer: study design, cohort characteristics, and feasibility of the St. Jude Lifetime Cohort study. Pediatr. Blood Cancer 56, 825-836 (2011).
12. Oeffinger KC, Eshelman DA, Tomlinson GE et al. Programs for adult survivors of childhood cancer. J. Clin. Oncol. 16, 2864-2867 (1998).

13. Harrop JP, Dean JA, Paskett ED. Cancer survivorship research: a review of the literature and summary of current NCI-designated cancer center projects. Cancer Epidemiol. Biomarkers Prev. 20, 2042-2047 (2011).

14. Haynes B. Barriers and bridges to evidence based clinical practice. BMJ317, 273-276 (1998).

15. Institute of Medicine. Clinical Practice Guidelines We Can Trust. The National Academies Press, Washington, DC, USA (2011).

16. Kremer LC, Mulder RL, Oeffinger KC et al. A worldwide collaboration to harmonize guidelines for the long-term follow-up of childhood and young adult cancer survivors: a report from the International Late Effects of Childhood Cancer Guideline Harmonization Group. Pediatr. Blood Cancer 60, 543-549 (2013).

\section{Websites}

101. Children's Oncology Group. Long-term follow-up guidelines for survivors of childhood, adolescent, and young adult cancers. Version 3.0 (2008). www.survivorshipguidelines.org

102. Dutch Childhood Oncology Group. Richtlijn follow-up na kinderkanker meer dan 5 jaar na diagnose. SKION, Den Haag/Amsterdam, The Netherlands (2010). [In Dutch] www.skion.nl

103. Scottish Intercollegiate Guidelines Network. Long term follow up of survivors of childhood cancer. A national clinical guideline (2004). www.sign.ac.uk

104. UK Children's Cancer Study Group Late Effects Group. Therapy based long term follow up practice statement (2011). www.cclg.org.uk 